\title{
Efecto de un inhibidor tirosina kinasa (imatinib) en pacientes con tumores estromales gastrointestinales metastásicos. Experiencia preliminar
}

\author{
Marcelo $\mathbf{G}$ arrido $S^{1}$, Mónica Moncada $\mathbf{M}^{1}$, \\ Grace Tapia $N^{1}, G$ onzalo Méndez $0^{2}$, Héctor $G$ alindo $A^{1}$, \\ Alvaro Huete $G^{3}$, Luis Ibáñez $A^{4}$, Sergio G onzález ${ }^{2}$, \\ Manuel Alvarez $\mathbf{Z}^{1}$. \\ Treatment of metastatic \\ gastrointestinal stromal tumors \\ with Imatinib. Report of four cases
}

Gastrointestinal stromal tumors (GIST) have mutations of the tyrosine kinase receptor. When they are localized, the treatment of choice is surgical excision, but advanced tumors have a limited response to chemo or radiotherapy. Imatinib (STI571 or Glivec ${ }^{\circledR}$ ) is a selective inhibitor or tyrosine kinase proteins that has been used successfully in the treatment of advanced GIST. We report four patients (two women) with a metastatic GIST that were treated with Imatinib $400 \mathrm{mg}$ day and followed for 40 months. The disease tumor stabilized in three patients and in one it had an initial reduction and progressed at the end of follow up. Therefore Imatinib can be a therapeutic alternative in patients with metastatic GIST (Rev Méd Chile 2007; 135: 1327-32).

(Key words: Antineoplastic agents; Imatinib mesylate; Protein kinase inhibitors)

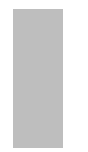

Recibido el 29 de septiembre, 2006. Aceptado el 27 de febrero, 2007.

${ }^{1}$ Departamento de Hematología-Oncología, ${ }^{2}$ Departamento de Anatomía Patológica, ${ }^{3}$ Departamento de Radiología, ${ }^{4}$ Departamento de Cirugía Digestiva, Centro de Cáncer, Pontificia Universidad Católica de Chile, Santiago de Chile.

Tos tumores estromales gastrointestinales L(GIST) representan 5\% de los sarcomas y son preferentemente gástricos (70\%). Presentan características histológicas, inmunohistoquímicas y ge-

Correspondencia a: Dr. Manuel Alvarez Z. Centro de Cáncer, Pontificia Universidad Católica de Chile. Diagonal Paraguay 319, Santiago, Chile. Fono: 56-2-3546919. Fax: 56-2-2472327. E mail: malvarez@med.puc.cl néticas propias ${ }^{1}$, distintas de leiomiomas, leiomiosarcomas y schwanomas.

En Estados Unidos de Norteamérica se estiman 150 nuevos casos al año ${ }^{2}$, en Chile representan $0,86 \%$ a $4 \%$ de las neoplasias gástricas ${ }^{3}$.

Los GIST se originan en las células intersticiales de Cajal y expresan en su superficie CD34 y CD117 (KIT).

La proteína KIT de transmembrana presente en células progenitoras hematopoyéticas, mastocitos, células germinales y las células intersticiales de 
Cajal $^{4}$ tiene $145 \mathrm{kD}$ y pertenece a la familia de receptores tirosina kinasa, codificada por el protooncogén c-kit ubicado en el cromosoma 4q11-q12.

El 85\% de los GIST presentan una mutación activante de la proteína KIT, que en 50\%-70\% de los casos es en el exón 115. La consecuencia es una proliferación celular descontrolada y resistencia a la apoptosis ${ }^{6}$. También se han detectado mutaciones en el receptor del factor de crecimiento derivado de plaquetas tipo A (PDGFRA) ${ }^{1}$. El estudio histopatológico permite certificar el diagnóstico, el subtipo celular, determinar la expresión de CD117 por inmunohistoquímica y determinar predictores de malignidad, como el recuento mitótico y el tamaño tumoral ${ }^{4}$.

El tratamiento estándar de los GIST localizados es la resección en bloque del tumor ${ }^{7}$. Los GIST irresecables o metastásicos son una enfermedad fatal, con tasas de respuesta entre $10 \%$ y $20 \%$ con quimioterapia y radioterapia.

Imatinib es un agente antineoplásico oral derivado de 2-fenilaminopirimidina, con un blanco molecular dirigido a un gnupo de tirosina kinasas: en KIT, BCR-ABL, ABL y PDGFRA. Actúa como un inhibidor competitivo del ATP que resulta en la inhibición de la proliferación celular y la restauración de la apoptosis ${ }^{8}$ (Figura 1). En ensayos clínicos imatinib ha demostrado efectividad en el tratamiento de leucemia mieloide crónica (LMC) y en GIST, fue aprobado por la Administración de Alimentos y Drogas de los Estados Unidos de Norteamérica (FDA) en LMC refractaria a interferón en mayo de $2001^{9}$ y en GIST en febrero de $2002^{10}$. Su uso clínico aumentó considerablemente desde la publicación, en abril de 2001, de un paciente con GIST metastásico que mostró rápida y sostenida respuesta por más de 18 meses $^{11}$.

Nuestro objetivo es mostrar la respuesta y tolerancia de los primeros pacientes con seguimiento de 40 meses con GIST metastásico tratados en el Centro del Cáncer de la Pontificia Universidad Católica de Chile desde julio de 2001.

\section{CASOS CLÍNICOS}

Entre los meses de julio y septiembre de 2001, cuatro pacientes con diagnóstico de GIST metastásico, confirmado por histología e inmunohistoquímica (positividad para CD117), iniciaron terapia

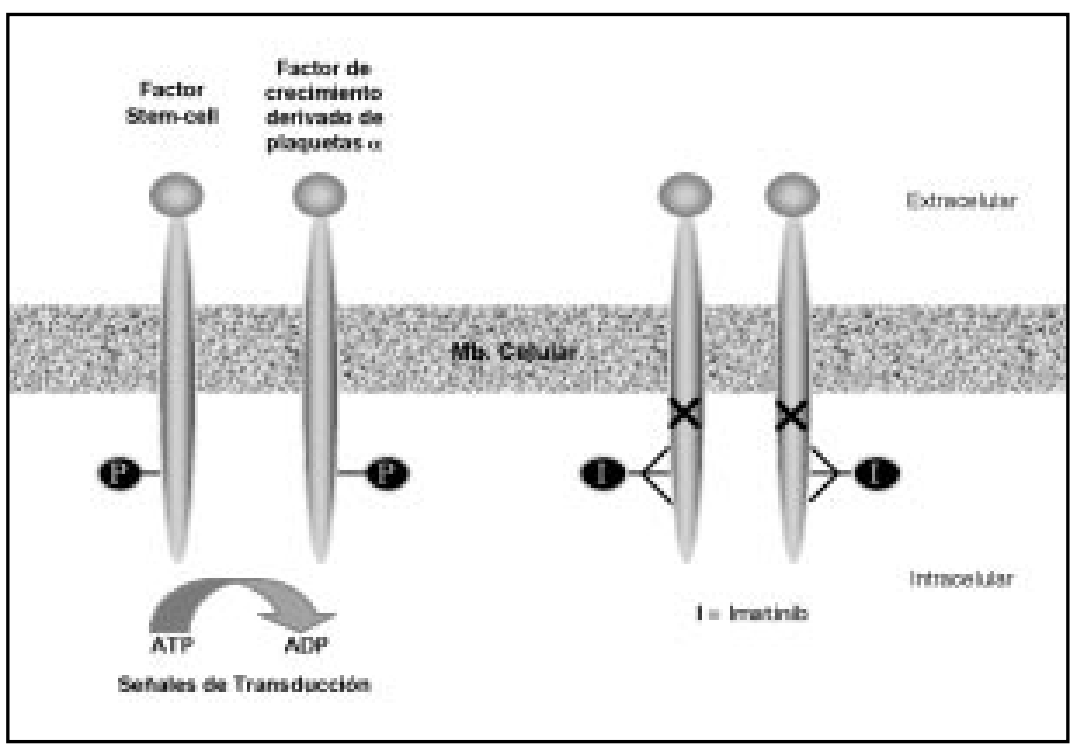

Figura 1. Esquema del mecanismo de acción molecular de C-KIT y el inhibidor de tirosin kinasa Imatinib. A la izquierda se muestra la activación de c-kit normal por la unión de su ligando factor de stem-cell o factor de crecimiento denivado de plaquetas $\alpha$, lo que promueve señales de transducción y proliferación celular. A la derecha se observa la función anormal de c-kit por mutación yuxtamembrana de GIST (exón 11), que produce activación constitutiva independiente de su ligando; esto es inhibido por la unión de Imatinib, bloqueando en forma selectiva la proliferación celular. 
con imatinib $400 \mathrm{mg} /$ día, completando un seguimiento de 40 meses.

Se registró las características clínicas y se realizó inicialmente una tomografía axial computada (TAC) de tórax, abdomen y pelvis. En el seguimiento se realizó evaluación clínica; TAC de tórax, abdomen y pelvis cada 3 meses y exámenes de laboratorio semanales (hemograma, perfil bioquímico y hepático) los 2 primeros meses, luego bisemanal y posteriormente mensual. Se registró la capacidad funcional (ECOG) y la toxicidad según criterios de la OMS.

Se estudió la histología a partir de tejidos fijados en formalina al 10\% e incluidos en parafina. Se efectuaron cortes de $5 \mu \mathrm{m}$ y se realizó tinción con hematoxilina-eosina e inmunohistoquímica para CD117 con anticuerpo policlonal anti c-kit humano C-19 (Santa Cruz Biotechnology, Santa Cruz, CA) (Figura 2).

Caso 1. Mujer de 49 años, operada en 1996 por un leiomiosarcoma. En diciembre de 2000 presentó múltiples lesiones metastásicas hepáticas y peritoneales, recibió 3 ciclos de quimioterapia, con enfermedad estable. En mayo de 2001 se realizó revisión de biopsia, diagnosticándose un GIST,
CD117 positivo. En la TAC de abdomen y pelvis de 19 de julio de 2001, se evidenciaron múltiples lesiones focales hepáticas, hasta de $11 \mathrm{~cm}$ de diámetro y múltiples lesiones en cavidad abdómino-pélvica, la mayor de $9 \mathrm{~cm}$ de diámetro. Tenía un ECOG estatus grado 3. El 30 de julio de 2001 inició imatinib con desaparición del dolor abdominal y de las masas palpables. La evaluación al tercer mes con imágenes mostró reducción de las lesiones hepáticas y abdómino-pélvicas mayor a $50 \%$. Las siguientes evaluaciones, hasta 40 meses, mostraron enfermedad estable.

Caso 2. Hombre de 67 años, con adenocarcinoma gástrico con metástasis hepáticas desde el 21 de junio de 2001. La revisión de biopsia mostró un GIST, CD117 positivo. En la TAC de abdomen y pelvis del $1^{0}$ de agosto de 2001, se observó una masa en la pared posterior del estómago a nivel de la curvatura menor de 13 x 11,5 cm y lesiones hepáticas múltiples. Tenía ECOG estatus grado 3. El 2 de agosto de 2001 inició imatinib, presentando pérdida de la sensación de plenitud gástrica, recuperación de su disfagia lógica y aumento de peso. La evaluación al tercer mes mostró una reducción de $50 \%$ de la lesión gástrica, mante-

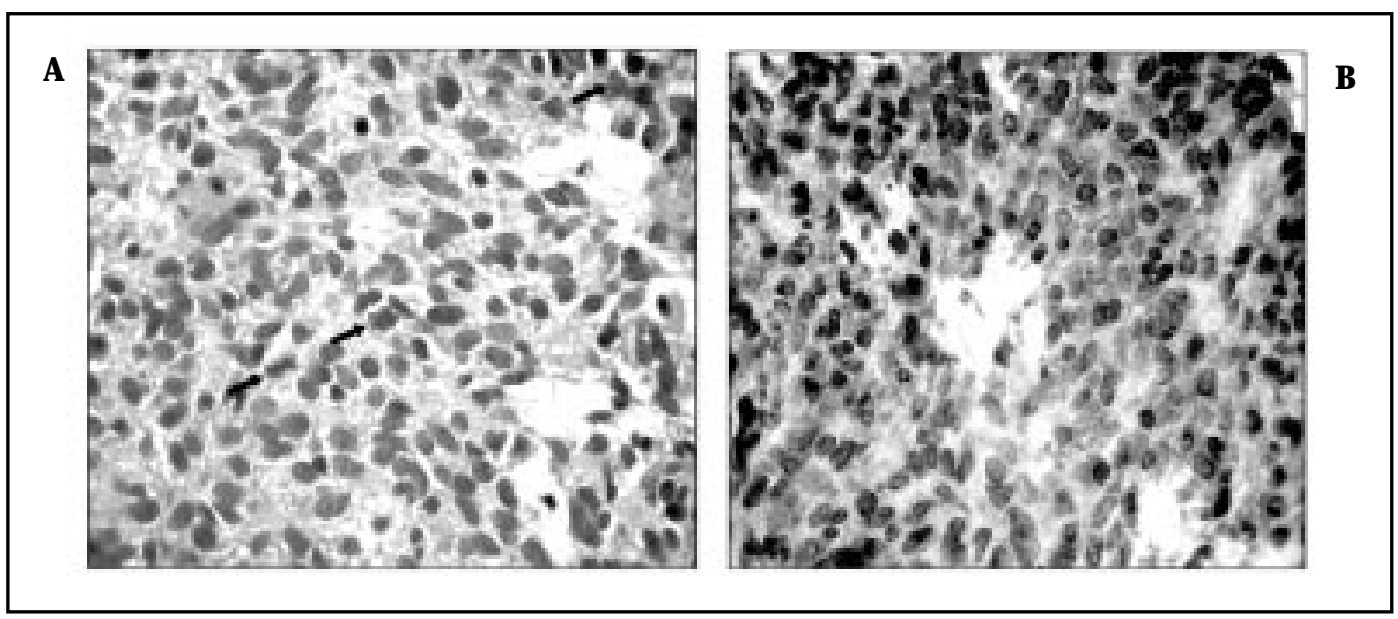

Figura 2. Histopatología e inmunohistoquímica del tumor de un paciente con GIST. A) Microfotografía de corte histológico teñido con hematoxilina-eosina ( $400 \mathrm{x}$ ), que muestra los signos de malignidad del tumor estromal gástrico. Se observa un área con células tumorales fusadas de límites citoplasmáticos poco definidos, con moderado pleomorfismo nuclear, sobreposición de núcleos que indica mayor densidad celular y algunos nucléolos prominentes. En el campo, se reconocen tres figuras mitóticas. B) Microfotografía de corte histológico del tumor con tinción inmunohistoquímica con anticuerpo anti c-kit humano (CD117). Se observa positividad intensa, café rojiza, en el citoplasma de las células tumorales. 
niendo enfermedad estable en evaluaciones posteriores (Figura 3A y 3B) y progresión diagnosticada al final del seguimiento de 40 meses.

Caso 3. Mujer de 60 años operada en marzo de 2001 con diagnóstico de un sarcoma estromal intestinal de yeyuno $(2 \times 1,5 \times 1,3 \mathrm{~cm})$, con metásta- sis pelviana $(14 \mathrm{x} 11 \mathrm{x} 6 \mathrm{~cm})$ y hepáticas, $\mathrm{CD} 117$ positivo. Recibió 3 ciclos de quimioterapia con progresión de enfermedad metastásica hepática, con ECOG estatus grado 2. Inició imatinib el 13 de agosto de 2001. La evaluación a los 3 meses mostró enfermedad metastásica hepática estable, sin aparición de nuevas lesiones. Se mantuvo con

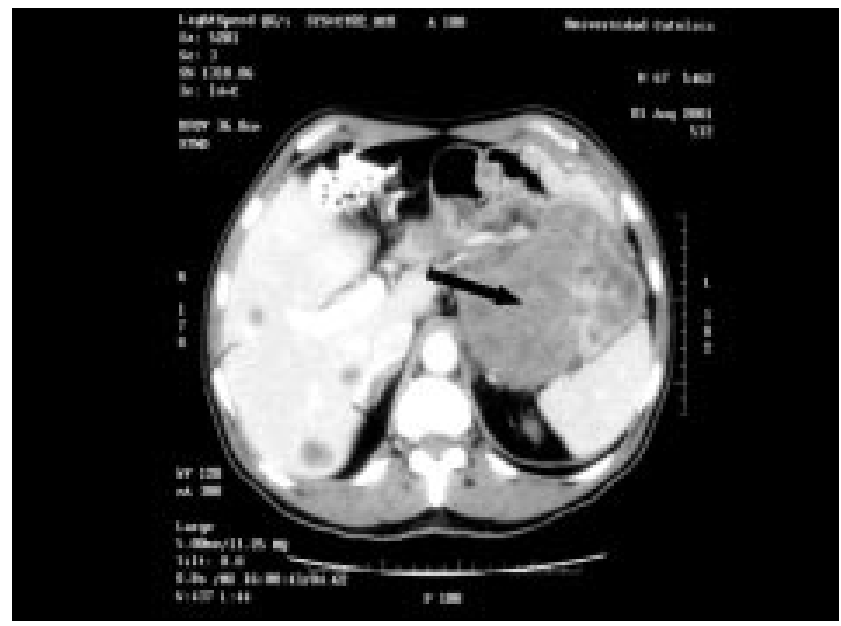

Figura 3A. Imagen de TAC de paciente con sarcoma estromal gástrico previo a inicio de terapia con Imatinib. TAC helicoidal de abdomen con uso de contraste E.V. a la altura del hilio esplénico demuestra una masa sólida, exocentérica, dependiendo del fondo gástrico de $9 \mathrm{~cm}$ de diámetro mayor. Además se observan 3 lesiones focales hepáticas sólidas, con refuerzo anular periférico que corresponden a metástasis hepáticas.

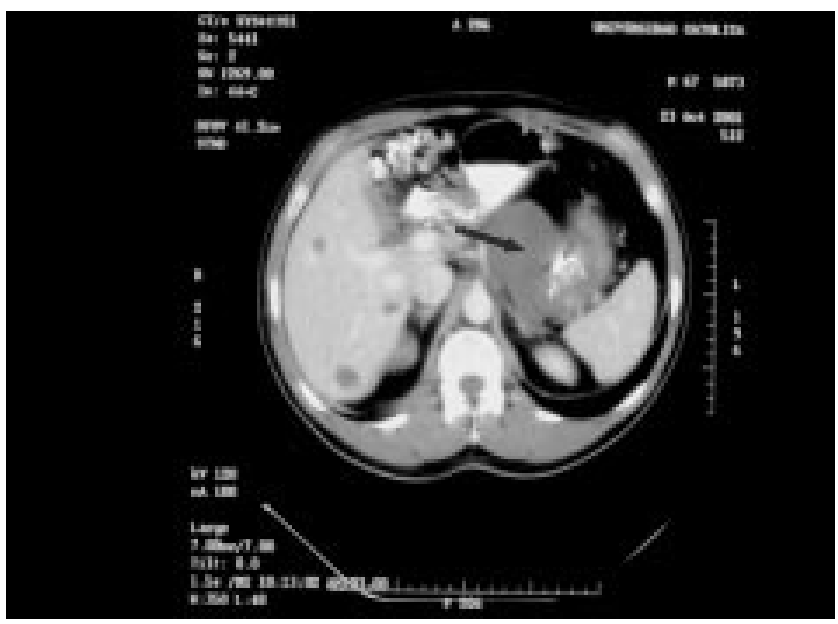

Figura 3B. Imagen de TAC de paciente con sarcoma estromal gástrico, 10 semanas después de iniciado tratamiento con Imatinib. Control tomográfico contrastado a las 10 semanas en un nivel similar demuestra marcada reducción en tamaño de masa gástrica, alcanzando $6 \mathrm{~cm}$ de diámetro mayor. Nótese además reducción en la impregnación del tumor compatible con necrosis. Las metástasis hepáticas también presentan disminución de tamaño y de captación de contraste. 
enfermedad estable en evaluaciones clínicas y de imágenes hasta 40 meses de seguimiento.

Caso 4. Hombre de 47 años operado en 1995. La revisión de biopsia confirmó un GIST, CD117 positivo. En 1999 se diagnosticaron metástasis hepáticas. TAC abdomen y pelvis inicial mostró hepatomegalia hasta nivel de la pelvis, secundaria a múltiples metástasis, la mayor en lóbulo hepático izquierdo de 28,8x19,9 cm y ascitis. El paciente presentó hiperbilirrubinemia, anasarca y ECOG estatus grado 3. El 8 de septiembre de 2001 inició imatinib. La evaluación a los 3 y 6 meses mostró enfermedad estable. Clínicamente el paciente presentó mejoría, con leve disminución de la hepatomegalia, sin anasarca, sólo con leve edema distal de extremidades inferiores, con regresión de hiperbilimubinemia y mejoría de capacidad funcional. Mantuvo enfermedad estable en evaluaciones hasta 40 meses de seguimiento.

Los efectos adversos observados fueron grado 1 y 2: neutropenia transitoria, anemia, edema periorbitario y náuseas. Un paciente presentó dispepsia manejada con la administración de las cápsulas junto a los alimentos, con buena respuesta. Otro paciente presentó dermatitis grado 2, controlada con antihistamínicos, corticoides orales en bajas dosis y corticoides tópicos. No hubo otros efectos adversos.

\section{DisCUSIÓN}

Los pacientes con GIST debutan con enfermedad metastásica en $50 \%$ de los casos, con una sobrevida mediana de 20 meses $^{5,12}$. En los 4 pacientes reportados no hubo muertes durante el periodo de seguimiento de 40 meses.

Imatinib presenta una alta tasa de respuesta en los pacientes con GIST, que puede evidenciarse en semanas si se evalúa por PET/CT y en meses al evaluarla por TAC; en nuestros pacientes se logró control de enfermedad en todos los casos y

\section{REFERENCIAS}

1. Corless CL, Fietcher JA, Heinrich MC. Biology of gastrointestinal stromal tumors. J Clin Oncol 2004; 22: 3813-25. respuesta objetiva en 50\%; estos resultados concuerdan con las tasas de respuesta publicadas ${ }^{13}$.

En estos pacientes utilizamos imatinib $400 \mathrm{mg} /$ día, la dosis estándar en el tratamiento inicial de los GIST. Dosis de $800 \mathrm{mg}$ muestran mejor sobrevida libre de progresión, pero no estadísticamente significativa al comparanla a dosis de $400 \mathrm{mg}^{13}$.

La duración óptima del tratamiento con imatinib no está definida ${ }^{13}$. En nuestra serie la dosis se mantuvo hasta la progresión, debido a que la interrupción del tratamiento después de un año presenta $21 \%$ de recaída ${ }^{14}$.

La terapia neoadyuvante se ha comunicado en casos y series clínicas. Aquellos pacientes con enfermedad estable previa a la cirugía tienen sobrevida global a 20 meses de $95 \%{ }^{15}$. En estos pacientes la enfermedad era irresecable y no se consideró su resecabilidad posterior a la respuesta con Imatinib.

La resistencia secundaria a imatinib se ha estudiado en pacientes con LMC. Los mecanismos involucrados son múltiples, los más importantes son mutaciones puntuales en el dominio kinasa de BCR-ABL ${ }^{16}$. En GIST se han detectado casos de resistencia a la terapia, debido a distintos sitios de mutación de exones del C-KIT y una variedad de delecciones y substituciones ${ }^{8,17}$.

El desarrollo de resistencia a imatinib es un potencial riesgo en los pacientes con GIST, pero surgen nuevas alternativas con fármacos como dasatinib, nilotinib y SU11248; los dos primeros disponibles para uso clínico en $\mathrm{LMC}^{18,19}$ y el tercero probado en líneas celulares y pacientes con GIST resistentes a imatinib ${ }^{20}$. Los pacientes con GIST irresecables y metastásicos no contaban con tratamiento sistémico efectivo; con el uso de Imatinib y los nuevos inhibidores de tirosina kinasa se amplían las alternativas terapéuticas en esta enfermedad.

Los resultados publicados y lo observado en nuestra serie han establecido a imatinib como a una alternativa que cambia paradigmas de tratamientos sobre blancos moleculares específicos en oncología.

2. Pidhorecky I, Cheney RT, Kraybill WG, Gibbs JF. Gastrointestinal stromal tumors: current diagnosis, biologic behavior, and management. Ann Surg Oncol 2000; 7: 705-12.

3. Llorens P, NaKamura K. Resultados del examen 
masivo del cáncer gástrico en Chile. JICA 1995; 143-55.

4. Graadt Van Roggen JF, Van Velthuysen ML, HogenDOORN PC. The histopathological differential diagnosis of gastrointestinal stromal tumours. J Clin Pathol 2001; 54: 96-102.

5. Connowy EM, GafFney E, Reynolds JV. Gastrointestinal stromal tumours. Br J Surg 2003; 90: 1178-86.

6. Hirota S, Isozaki $\mathrm{K}$, Moriyama $\mathrm{Y}$, Hashimoto $\mathrm{K}$, Nishida T, IsHiguRo $S$ ET AL. Gain-of-function mutations of c-kit in human gastrointestinal stromal tumors. Science 1998; 279: 577-80.

7. Joensuu H, Fietcher C, Dimitrujevic S, Silberman S, RoBerTs P, DeMETRI G. Management of malignant gastrointestinal stromal tumours. Lancet Oncol 2002; 3: 655-64.

8. Savage DG, Antman KH. Imatinib Mesylate - A New Oral Targeted Therapy. N Engl J Med 2002; 346: 683-93.

9. Druker BJ, Talpaz M, Resta DJ, Peng B, Buchdunger E, FoRD JM ET AL. Efficacy and safety of a specific inhibitor of the BCR-ABL tyrosine kinase in chronic myeloid leukemia. N Engl J Med 2001; 344: 1031-7.

10. Dagher R, Cohen M, Wiwams G, Rothmann $M$, GobBuRu J, RobBie G et al. Approval summary: Imatinib mesylate in the treatment of metastatic and/or unresectable malignant gastrointestinal stromal tumors. Clin Cancer Res 2002; 8: 3034-8.

11. Joensuu H, Roberts PJ, Sarlomo-Rikala M, Andersson LC, Tervahartiala P, Tuveson D et al. Effect of the tyrosine kinase inhibitor STI571 in a patient with a metastatic gastrointestinal stromal tumor. $\mathrm{N}$ Engl J Med 2001; 344: 1052-6.

12. Dematteo RP, Lewis JJ, Leung D, Mudan SS, WooDRUFF JM, BRENNAN MF. Two hundred gastrointestinal stromal tumors: recurrence patterns and prognostic factors for survival. Ann Surg 2000; 23: $51-8$.

13. Verweij J, Casal PG, Zalcberg J, Lecesne A, ReicharDT P, Blay JY ET al. Progression-free survival in gastrointestinal stromal tumours with high-dose Imatinib: randomised trial. Lancet 2004; 364: 1127-34.

14. Blay Jy, Berthaud P, Perol D, Ray-Coquard I, Bui B, DufFaud F ET aL. Continuous vs intermittent Imatinib treatment in advanced GIST after one year: A prospective randomized phase III trial of the French Sarcoma Group. Proc Am Soc Clin Oncol 2004; 23 (Abstr 9006).

15. Raut CP, Posner M, Desai J, Morgan JA, George S, ZAHRIEH D ET AL. Surgical management of advanced gastrointestinal stromal tumors after treatment with targeted systemic therapy using kinase inhibitors. J Clin Oncol 2006; 24: 2325-31.

16. Cortés J, Kantarian $\mathrm{H}$. New targeted approaches in chronic myeloid leukemia. J Clin Oncol 2005; 23: 6316-24.

17. WeISBERg E, GrifFin JD. Resistance to Imatinib (Glivec): update on clinical mechanisms. Drug Resist Updat 2003; 6: 231-8.

18. Talpaz M, Shah NP, Kantarian H, Donato N, Nicol J, Paquette R et al. Dasatinib in Imatinib-resistant Philadelphia chromosome-positive leukemias. N Engl J Med 2006; 354: 2531-41.

19. Kantarjian H, Giles F, Wunderle L, Bhalla K, O'BRIEN S, Wassmann B ET aL. Nilotinib in Imatinibresistant CML and Philadelphia chromosomepositive ALL. N Engl J Med 2006; 354: 2542-2551.

20. Prenen H, Cools J, Mentens N, Folens C, Sciot R, SCHOFFSKI P ET AL. Efficacy of the kinase inhibitor SU11248 against gastrointestinal stromal tumor mutants refractory to Imatinib mesylate. Clin Cancer Res 2006; 12: 2622-7. 